\title{
Hybridizing Grid Search and Support Vector Regression to Predict the Compressive Strength of Fly Ash Concrete
}

\author{
Fei Tang, ${ }^{1}$ Yanqi $W u \mathbb{D}^{2},{ }^{2}$ and Yisong Zhou ${ }^{3}$ \\ ${ }^{1}$ School of Architectural Engineering, Xinyang Vocational and Technical College, Xinyang 464000, China \\ ${ }^{2}$ School of Civil Engineering, Southeast University, Nanjing 211189, China \\ ${ }^{3}$ School of Civil Engineering, Xinyang College, Xinyang 464000, China \\ Correspondence should be addressed to Yanqi Wu; yanqiwu@seu.edu.cn
}

Received 23 December 2021; Accepted 8 February 2022; Published 23 February 2022

Academic Editor: Ravindran Gobinath

Copyright $\odot 2022$ Fei Tang et al. This is an open access article distributed under the Creative Commons Attribution License, which permits unrestricted use, distribution, and reproduction in any medium, provided the original work is properly cited.

\begin{abstract}
Support vector regression (SVR) has been applied to the prediction of mechanical properties of concrete, but the selection of its hyperparameters has been a key factor affecting the prediction accuracy. To this end, hybrid machine learning combines the SVR model and grid search (GS), namely, the GS-SVR model was proposed to predict the compressive strength of concrete and sensitivity analysis in this work. The hybrid model was trained and tested on a total of 98 datasets retrieved from literature, and the model performance was compared with the original SVR model under the same datasets. The obtained results in terms of $R$ of 0.981, MSE of 3.44, RMSE of 1.85, MAE of 1.17, and MAPE of 0.05 demonstrate that the GS-SVR model proposed can be a candidate method for compressive strength prediction in subsequent related studies. Additionally, a graphical user interface (GUI) was developed to conveniently provide some initial estimates of the outcomes before performing extensive laboratory or fieldwork. Finally, the effect of each variable on the compressive strength in a random environment was analyzed.
\end{abstract}

\section{Introduction}

As the most consumed material in the construction industry, cement has brought great convenience to the construction industry. But the bad news is that it also puts enormous pressure on the environment. Since the production and utilization of cement are accompanied by a large amount of greenhouse gas emissions, many scholars have started to focus on research related to mineral admixtures that can be used to replace cement [1-5]. Among them, the use of fly ash as an auxiliary cementitious material for the production of a large amount of fly ash concrete is one of the important ways to reduce environmental pollution and realize the resourcefulness of fly ash. Moreover, it is also an effective means for concrete producers to enhance and improve the performance of concrete in all aspects, reduce the use of cement, and lower the cost of concrete $[6,7]$. The incorporation of fly ash not only ensures the quality of concrete and reduces the cost of manufacturing concrete but also improves the compatibility, durability, and strength, thus becoming the most widely used alternative and receiving great attention [8].

The importance of concrete materials for the construction industry needs no more introduction [9-11]. Concrete is used as a construction material worldwide due to its various properties such as strength, durability, stiffness, and fire resistance. Among these properties, compressive strength is considered to be the most important one because it seriously affects the safety and durability of concrete members. Understanding the early behavior of concrete allows appropriate measures to be taken to avoid problems such as cracking and deformation of concrete members, formwork failure, and rework. In addition, early strength prediction and monitoring are important for assessing construction safety, determining structural maturity, and predicting later strength development. The main reason for different compressive strength values of concrete is that concrete is a nonhomogeneous material consisting of binders, aggregates, water, and admixtures. In such a complex mix, it is difficult to find or predict the compressive 
strength of concrete accurately. The compressive strength of concrete can be assessed in the laboratory by crushing a standard size cylinder or cube. However, such laboratory tests are supposed to be inefficient and uneconomical, as well as labor-intensive and time-consuming. Traditional methods of concrete strength prediction are mainly based on a statistical analysis of linear or nonlinear regression equations, but obtaining accurate equations is difficult and requires a great deal of skill and experience. Due to the large number of concrete ingredients, it seems difficult to establish an explicit equation between the compressive strength and each ingredient as a way to predict its compressive strength.

To address these issues, machine learning techniques are used to predict the compressive strength of concrete. In fact, with the development of artificial intelligence, various machine learning algorithms such as artificial neural network (ANN), support vector machines (SVM), random forest, and extreme learning machine (ELM) have been applied to predict the mechanical properties of concrete [12-23]. Ly et al. [24] employed an optimal deep neural network model on a database of 223 experimental data to predict the 28 days compressive strength of rubber concrete and achieved a high prediction accuracy of $R=0.9874$. Han et al. [25] utilized an optimized random forest approach on 1030 data samples collected from published literature to predict the compressive strength of high-performance concrete. Furqan et al. [26] used the ANN, SVM, and gene expression programming (GEP) on 300 datasets to predict the compressive strength of self-compacting concrete. Zhang et al. [27] employed nine machine learning models to predict the compressive strength of concrete at the age of 7 days and found that the nonlinear models had better predictive performance than the linear models. Khoa et al. [28] employed deep neural networks (DNNs) and ResNet models for compressive strength prediction of green fly ash-based geopolymer concrete. The results showed that ResNet is superior and indicated that these two machine learning methods can be useful for the mixed design of geopolymer concrete. From the published studies, it can be found that these machine learning algorithms outperform traditional empirical formulations by enabling the capture and mapping of multidimensional nonlinear relationships. It is possible to extract unknown relationships and data information between input and output variables [29].

However, these models also have limitations, and many models require parameter tuning to get better performance. For support vector regression (SVR), the selection of hyperparameters has a great impact on the accuracy of the prediction results. In other words, the prediction accuracy of a single SVR model is limited. To better understand and apply the SVR method, further exploration is still needed in this area using different datasets and optimization algorithms. For this reason, this study aims to propose a hybrid machine learning that combines the SVR model and grid search (GS), namely, the GS-SVR model, to achieve an accurate prediction of the compressive strength of fly ash concrete. Based on the model, the effect of random variation of individual variables on compressive strength is investigated as a reference and guide for concrete mix design and strength prediction.

\section{Methodology}

2.1. Support Vector Regression. The objective of the SVR is to find a linear regression equation that fits all sample points and minimizes the total variance of the sample points from this regression hyperplane. There is a sample training set $E=\left\{\left(x_{i}, y_{i}\right) \mid i=1,2, \ldots n\right\}, x_{i} \in R^{n}, y_{i} \in R$. A function $f\left(x_{i}\right)$ is probed on $R^{n}$, such that $y_{i}=f\left(x_{i}\right)$, and there is always a corresponding $y$-value for any input $x$. Such a function $f\left(x_{i}\right)$ is called a regression function, and $f\left(x_{i}\right)$ can be described as follows.

$$
f\left(x_{i}\right)=\omega \cdot \phi\left(x_{i}\right)+b,
$$

where $\omega \in R^{n}$ is the weight vector, $\phi\left(x_{i}\right)$ is a nonlinear mapping which serves to map the data from the space $R^{n}$ to the higher feature space, and $b$ is the bias. Equation (1) can be fitted to all sample points at precision $\varepsilon$.

$$
\left|y_{i}-\left[\omega \cdot \phi\left(x_{i}\right)+b\right]\right| \leq \varepsilon, \quad i=1,2, \ldots, n .
$$

Since there is a certain fitting error, the slack variables $\left(\xi_{i}, \xi_{i}{ }^{*}\right)$ and the penalty parameter $C$ are introduced. The regression fitting problem is changed to an optimization problem.

$$
\begin{aligned}
& \min \left(R\left(\omega, \xi_{i}, \xi_{i}^{*}\right)\right)=\frac{1}{2} \omega \cdot \omega+C \sum_{i=1}^{n}\left(\xi_{i}+\xi_{i}^{*}\right), \\
& \text { s.t. }\left\{\begin{array}{l}
y_{i}-\left[\omega \cdot \phi\left(x_{i}\right)+b\right] \leq \varepsilon+\xi_{i}, \\
\omega \cdot \phi\left(x_{i}\right)+b-y_{i} \leq \varepsilon+\xi_{i}^{*}, \\
\xi_{i}, \xi_{i}^{*} \geq 0 .
\end{array}\right.
\end{aligned}
$$

Using Lagrange multipliers for equation (3), the Lagrange function is introduced to obtain its dual form. 


$$
\begin{aligned}
& \max \left(W\left(\alpha, \alpha^{*}\right)=-\frac{1}{2} \sum_{i, j=1}^{n}\left(\alpha_{i}-\alpha_{i}^{*}\right)\left(\alpha_{j}-\alpha_{j}^{*}\right)\left[\phi\left(x_{i}\right) \cdot \phi\left(x_{j}\right)\right]-\sum_{i=1}^{n}\left(\alpha_{i}+\alpha_{i}^{*}\right) \varepsilon+\sum_{i=1}^{n}\left(\alpha_{i}-\alpha_{i}^{*}\right) y_{i},\right. \\
& \text { s.t. }\left\{\begin{array}{l}
\sum_{i=1}^{n}\left(\alpha_{i}-\alpha_{i}^{*}\right)=0, \\
0 \leq \alpha_{i}, \alpha_{i}^{*} \leq C, i=1,2, \ldots, n,
\end{array}\right. \\
& \omega=\sum_{i=1}^{n}\left(\alpha_{i}-\alpha_{i}^{*}\right) \phi\left(x_{i}\right) .
\end{aligned}
$$

The core of optimization at this point is to first determine the feature space and find the flattest function in that space that satisfies the conditions and then use that function to solve the nonlinear problem. For this reason, the kernel function $K\left(x_{i}, x_{j}\right), K\left(x_{i}, x_{j}\right)=\phi\left(x_{i}\right) \cdot \phi\left(x_{j}\right)$ is introduced. The regression fitting function at this point is

$$
f\left(x_{i}\right)=\sum_{i, j=1}^{n}\left(\alpha_{i}-\alpha_{i}^{*}\right) K\left(x_{i}, x_{j}\right)+b .
$$

There are many choices of kernel functions, and the commonly used RBF function (Figure 1) is chosen in this study [30].

2.2. Grid Search Method. It is well known that the identification results depend heavily on the selection of hyperparameters of the SVR model. Since the parameters are highly nonlinear, a large number of experiments are often required to determine the combination of parameters, such as the penalty parameter $C$ and the kernel parameter $g$. Although Lin et al. [31, 32] have done much extensive research to simplify the application of SVM, such as proposing LIBSVM, the selection of parameters $C$ and $g$ still depends on experience. Therefore, there is an urgent need to implement automatic tuning of parameters to obtain the optimal value once the parameters are entered. The grid search (GS) method can solve these problems. The GS method is to computationally evaluate the impact of each parameter combination on the model performance by iterating through all the candidate parameter choices in a loop to obtain the optimal combination of hyperparameters [33]. The flowchart of parameter selection is shown in Figure 2.

\section{Dataset Description}

3.1. Input and Output Variables. In this study, 98 sets of data were retrieved from the literature. Each dataset consisted of six constituents (cement, fly ash, water, super, plasticizer, coarse aggregate, and fine aggregate) and age as input variables and the compressive strength as output variable. The distribution of the input and output variables is shown in Figure 3, and the statistical characteristics of these variables are given in Table 1. It can be seen that the compressive strength varies greatly for different combinations of input variables. Additionally, Pearson correlation coefficients between input and output variables were plotted, as shown in Figure 4. Within the current dataset, linear correlation between any of the input and output variables is weak, which indicates a complex nonlinear relationship between compressive strength and these input parameters. Therefore, the relationship between compressive strength and these parameters is difficult to reflect by an explicit equation.

3.2. Performance Criteria. To describe and compare the performance of the models, five evaluation metrics, linear correlation coefficient $(R)$, mean squared error (MSE), mean root error (RMSE), mean absolute percentage error (MAPE), and mean absolute error (MAPE), were introduced [34]. These metrics are defined as follows.

$$
\begin{aligned}
R & =\frac{n \sum_{i=1}^{n}\left(y_{e} y_{p}\right)-\sum_{i=1}^{n} y_{e} \sum_{i=1}^{n} y_{p}}{\sqrt{\left[n\left(\sum_{i=1}^{n} y_{e}^{2}\right)-\left(\sum_{i=1}^{n} y_{e}\right)^{2}\right]\left[n\left(\sum_{i=1}^{n} y_{p}^{2}\right)-\left(\sum_{i=1}^{n} y_{p}\right)^{2}\right]}}, \\
\mathrm{MAE} & =\frac{1}{n} \sum_{i=1}^{n}\left|y_{e}-y_{p}\right|, \\
\mathrm{MSE} & =\frac{1}{n} \sum_{i=1}^{n}\left(y_{e}-y_{p}\right)^{2}, \\
\mathrm{RMSE} & =\sqrt{\frac{1}{n} \sum_{i=1}^{n}\left(y_{e}-y_{p}\right)^{2},} \\
\mathrm{MAPE} & =\frac{1}{n} \sum_{i=1}^{n}\left|\frac{y_{e}-y_{p}}{y_{e}}\right| \times 100 \%,
\end{aligned}
$$

where $n$ is the number of samples, $y_{e}$ is the experimental value of compressive strength, and $y_{p}$ is the predicted value. When $R$ is closer to 1 or the other four error indicators are closer to 0 , the prediction performance of the model is better.

\section{Model Performance}

Initially, GS is used for the selection of hyperparameters in the SVR. For the GS-SVR model, $C$ and $g$ are searched in an exponential grid of $\left[2^{-8}, 2^{8}\right]$ with step $2^{0.1}$. The evolution of the parameters is shown in Figure 5. The model is trained using 10-fold cross-validation. 


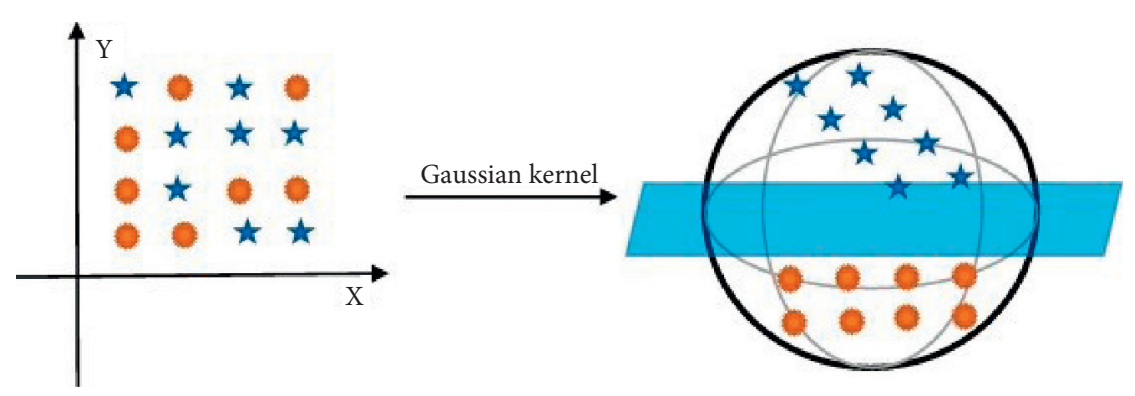

FIGURE 1: Schematic diagram of support vector machine and Gaussian kernel.

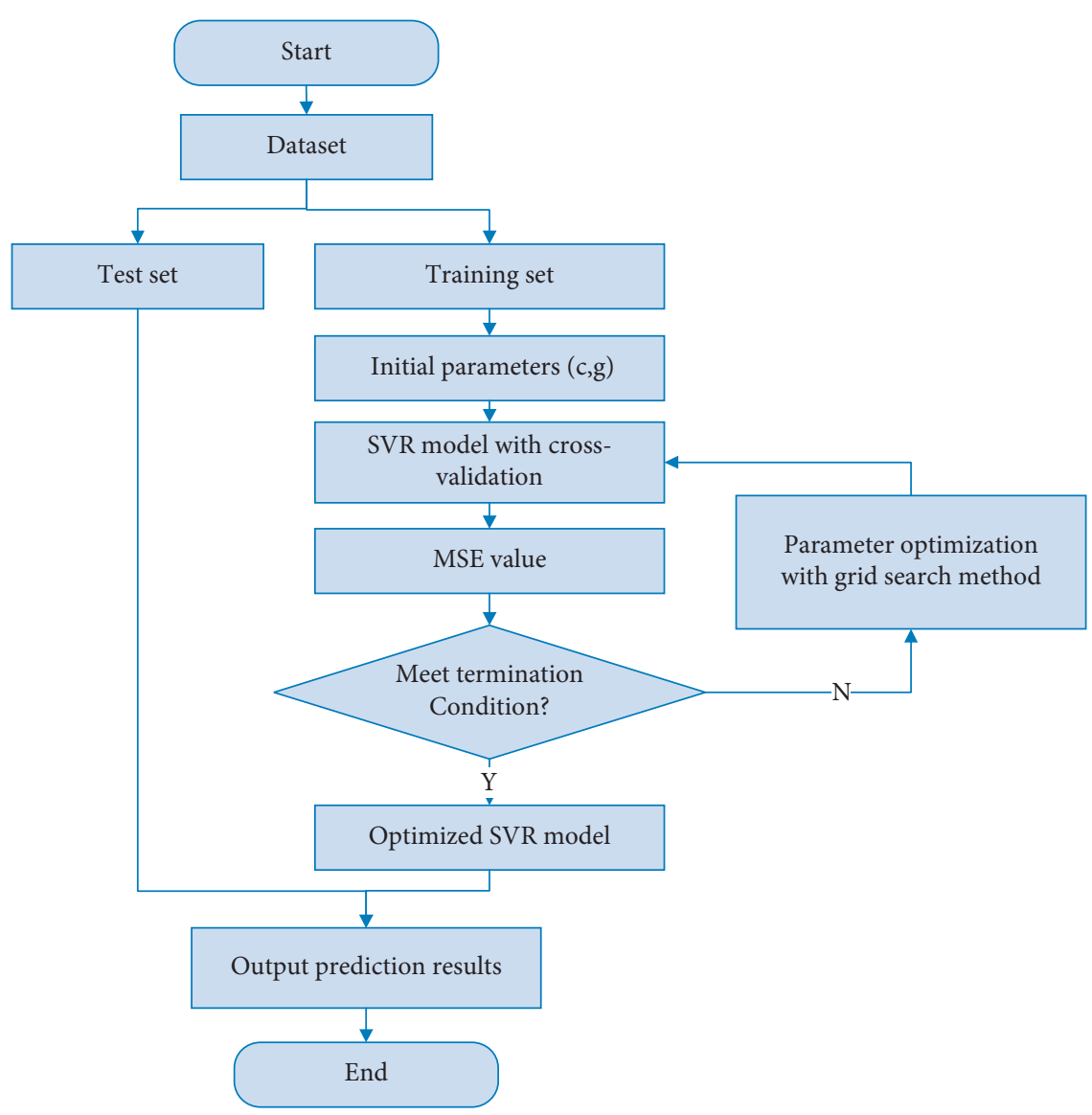

FIGURE 2: Flowchart of the SVR model using the GS method and cross-validation.

To highlight the superiority of the proposed method in this study, the original SVR model and GS-SVR model were performed on the same training and test set. The model results are shown in Figure 6. It can be clearly observed that compared with the SVR model, the GS-SVR model results have a closer distribution of data points along the diagonal, indicating that the predicted values match better with the experimental values, and the correlation coefficient $R$ exceeds 0.98 for both the training and test sets.

For comparison and evaluation purposes, Figure 7 shows the predicted and experimental values for the training and test sets in more detail. At each sample point, the predicted values agree well with the experimental values, demonstrating the accuracy and effectiveness of the GS-SVR model in capturing the complex nonlinear relationships between the seven input variables and the compressive strength. The error metrics for model training and test are shown in Figure 8. It can be clearly observed from both sets that the four error indicators of the GS-SVR model are smaller, further validating the accuracy and generalization capability of the proposed GS-SVR model.

\section{Sensitivity Analysis}

The model results in Section 4 show that the compressive strength depends on the input vector consisting of six ingredients and age. However, these variables have almost no deterministic values in a stochastic 


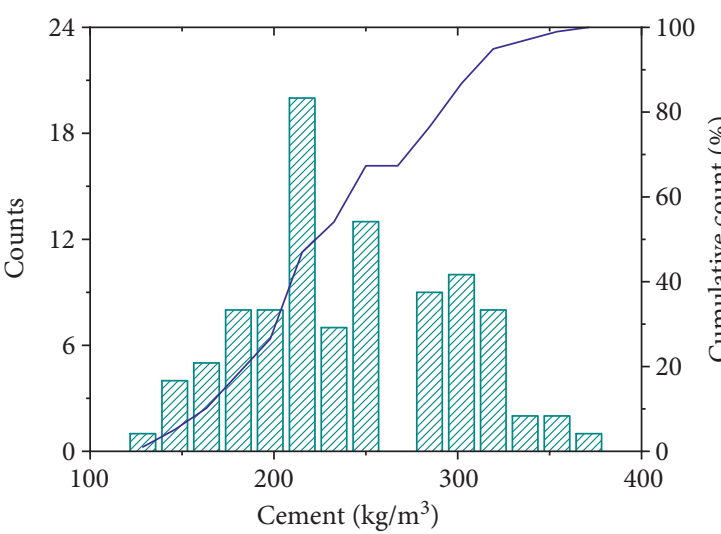

(a)

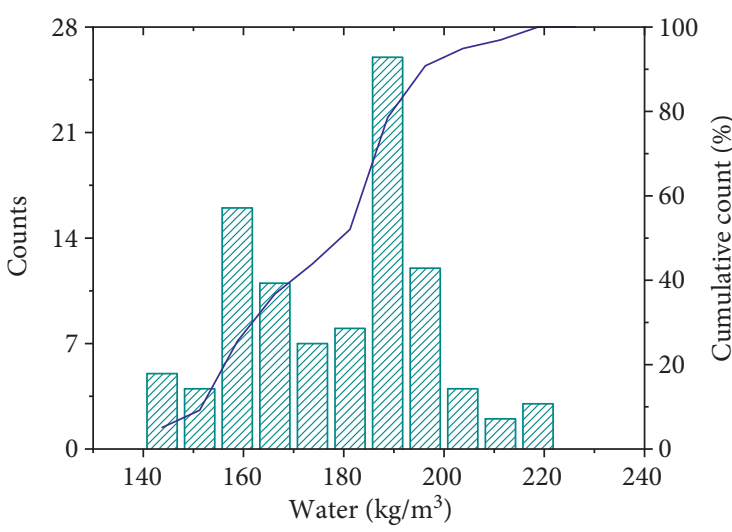

(c)

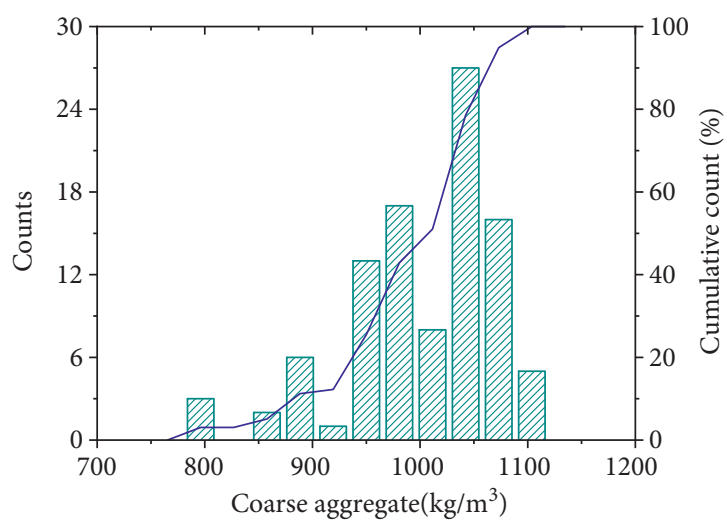

(e)

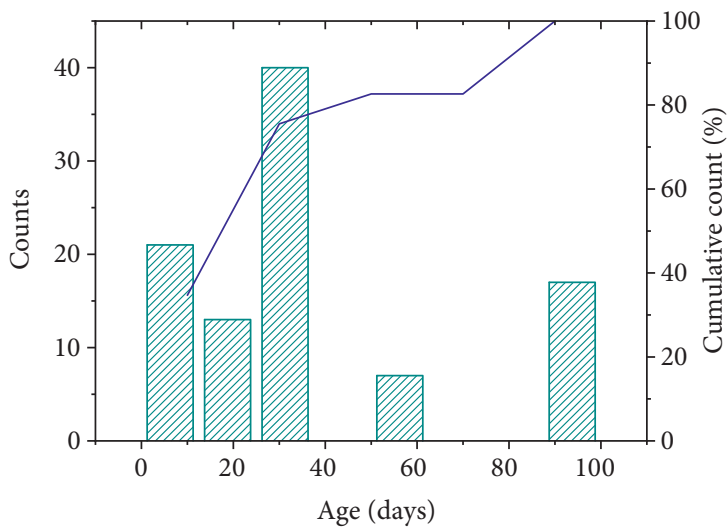

(g)

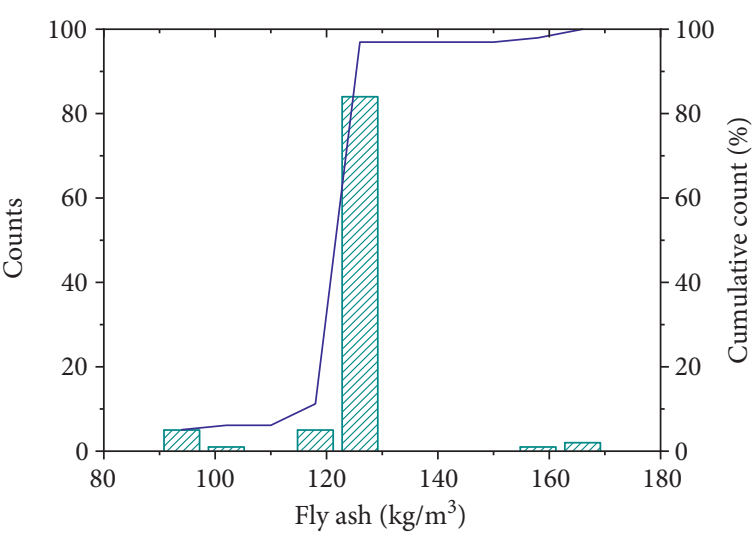

(b)

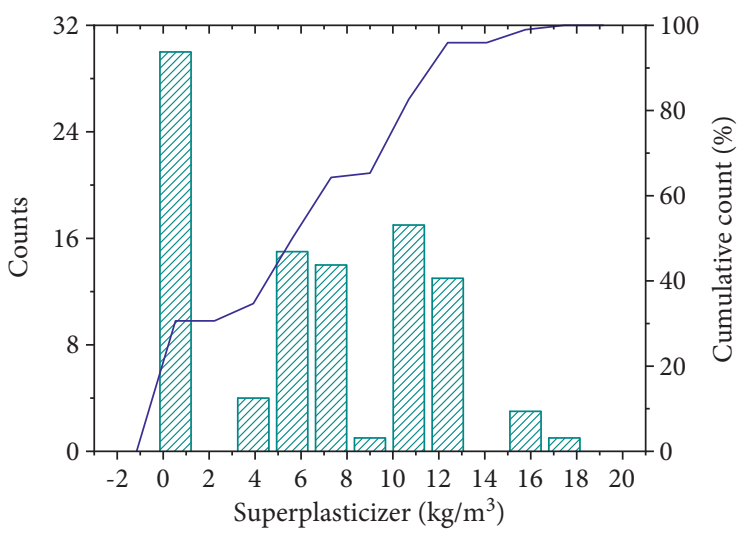

(d)

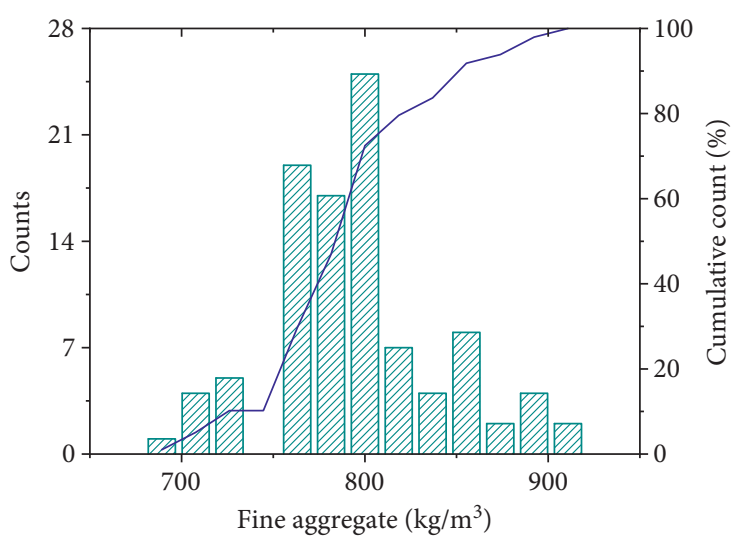

(f)

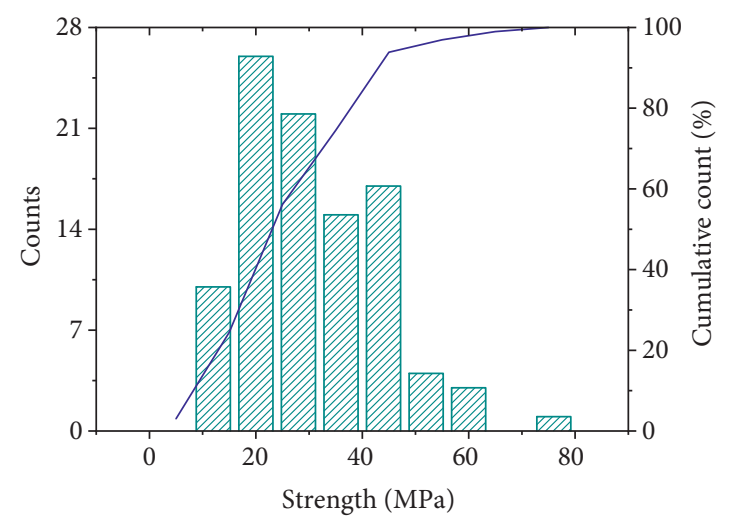

(h)

FIgURE 3: Distribution of input and output variables. 
TABLE 1: Statistical characteristics of variables.

\begin{tabular}{|c|c|c|c|c|c|c|c|c|c|}
\hline & & Cement & Fly ash & Water & Super plasticizer & Coarse aggregate & Fine aggregate & Age & $\begin{array}{l}\text { Compressive } \\
\text { strength }\end{array}$ \\
\hline \multirow{8}{*}{ Training set } & Unit & $\mathrm{kg} / \mathrm{m}^{3}$ & $\mathrm{~kg} / \mathrm{m}^{3}$ & $\mathrm{~kg} / \mathrm{m}^{3}$ & $\mathrm{~kg} / \mathrm{m}^{3}$ & $\mathrm{~kg} / \mathrm{m}^{3}$ & $\mathrm{~kg} / \mathrm{m}^{3}$ & $\mathrm{~d}$ & $\mathrm{MPa}$ \\
\hline & Count & 78 & 78 & 78 & 78 & 78 & 78 & 78 & 78 \\
\hline & Maximum & 376 & 163.3 & 216.7 & 18 & 1118 & 905.4 & 90 & 72.11 \\
\hline & Minimum & 136.1 & 92.1 & 141.1 & 0 & 801 & 700 & 3 & 9.49 \\
\hline & Standard deviation & 54.22 & 9.68 & 18.12 & 4.65 & 67.60 & 44.70 & 29.72 & 13.37 \\
\hline & Mean & 243.95 & 123.54 & 177.77 & 6.46 & 1010.62 & 800.34 & 34.06 & 31.85 \\
\hline & Skewness & 0.25 & -0.05 & -0.18 & -0.07 & -0.97 & 0.63 & 0.97 & 0.63 \\
\hline & Kurtosis & -0.75 & 9.44 & -0.81 & -0.88 & 0.79 & 0.23 & -0.40 & 0.02 \\
\hline \multirow{7}{*}{ Test set } & Count & 20 & 20 & 20 & 20 & 20 & 20 & 20 & 20 \\
\hline & Maximum & 349 & 168.3 & 220.5 & 16.1 & 1111.6 & 856.5 & 90 & 41.64 \\
\hline & Minimum & 144 & 95.7 & 158.2 & 0 & 801.1 & 687 & 3 & 9.55 \\
\hline & Standard deviation & 59.46 & 11.81 & 17.50 & 5.79 & 75.95 & 43.85 & 25.71 & 9.53 \\
\hline & Mean & 231.28 & 125.19 & 182.27 & 5.77 & 969.55 & 771.99 & 32.70 & 25.49 \\
\hline & Skewness & 0.46 & 1.78 & 0.51 & 0.34 & -0.29 & -0.17 & 1.52 & 0.21 \\
\hline & Kurtosis & -0.80 & 10.57 & -0.11 & -1.47 & -0.05 & -0.73 & 1.65 & -1.20 \\
\hline
\end{tabular}

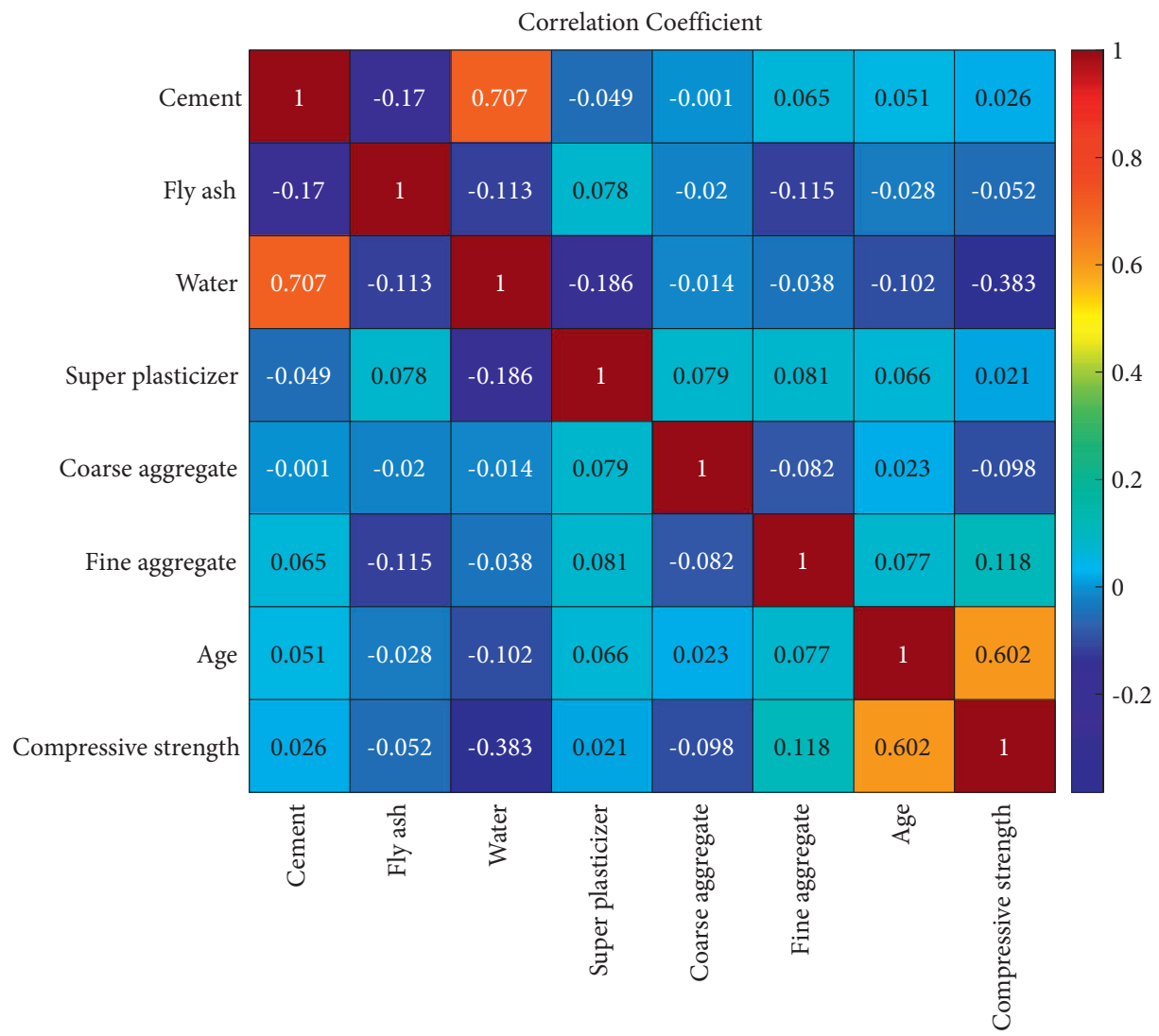

FIGURE 4: Correlation coefficient of the dataset.

environment. This uncertainty may cause the deviation of the predicted and actual values of the concrete compressive strength. Therefore, this section focuses on the effect of the random variation degree of each input variable on the compressive strength. According to the statistical characteristics of the dataset given in Table 1, a set of input vectors with deterministic configurations is given in Table 2 . The input variables are assumed to be at three different values of degree of stochasticity $S_{0}=(0.05,0.1,0.15)$. In each stochastic setting, $10^{4}$ samples were generated using MATLAB. To quantify and compare the degree of influence on the output variable, the sensitivity of the random input to the compressive strength is defined as follows $[35,36]$. 


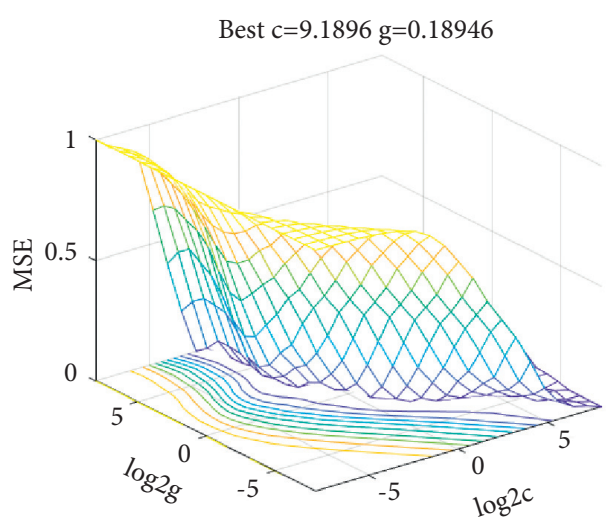

(a)

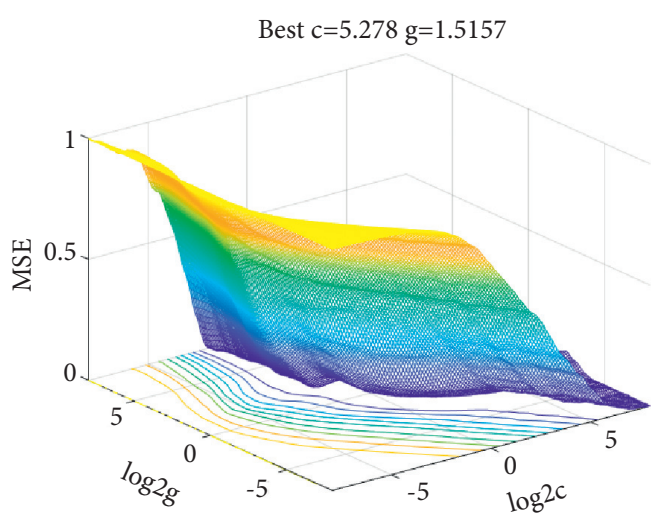

(b)

FIGURE 5: Hyperparameter selection results of the SVR model.

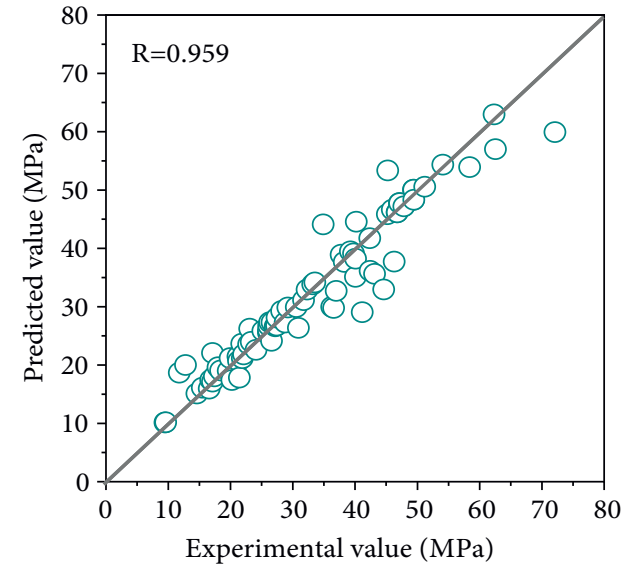

$\bigcirc$ Train

(a)

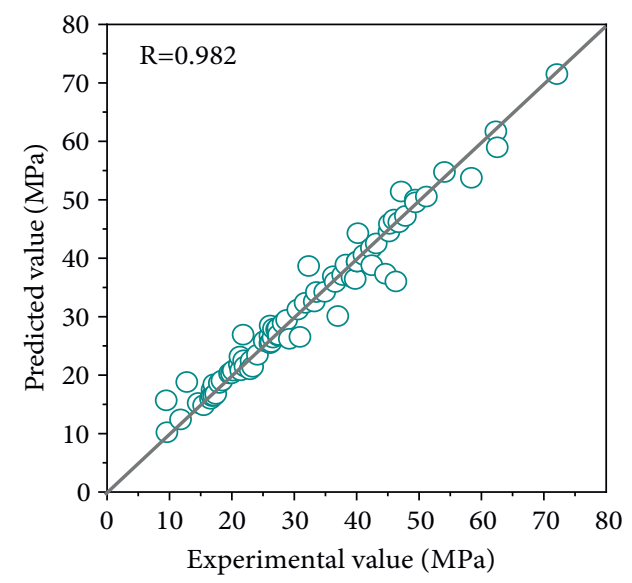

Train

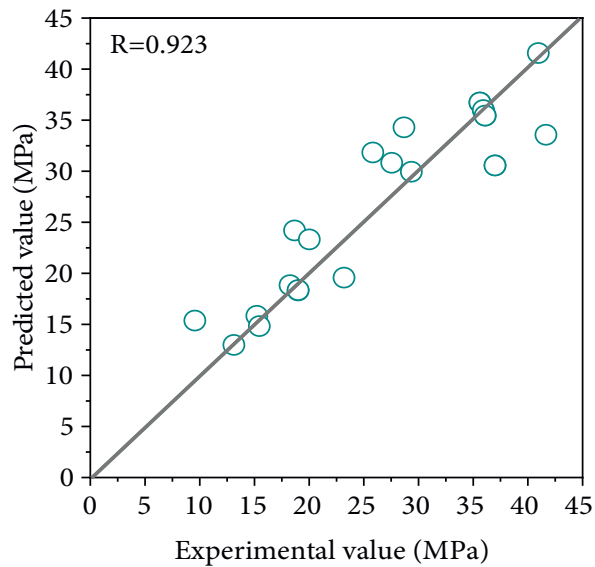

Train

(b)

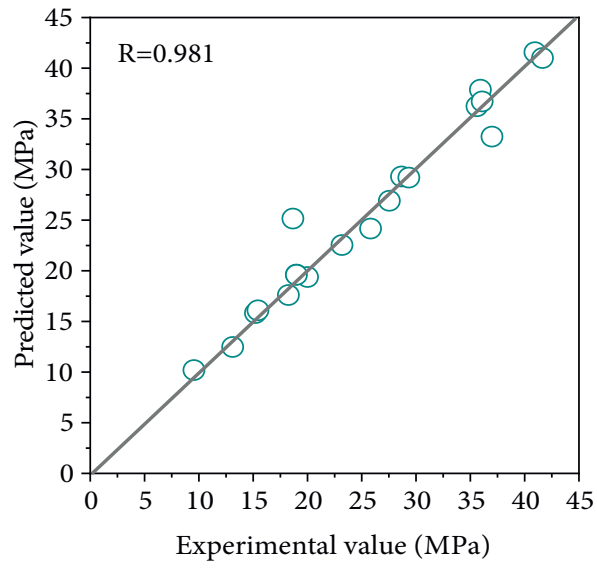

Train

(c)

(d)

FIGURE 6: Correlation between predicted and experimental values of two models. (a) SVR model. (b) GS-SVR model. 


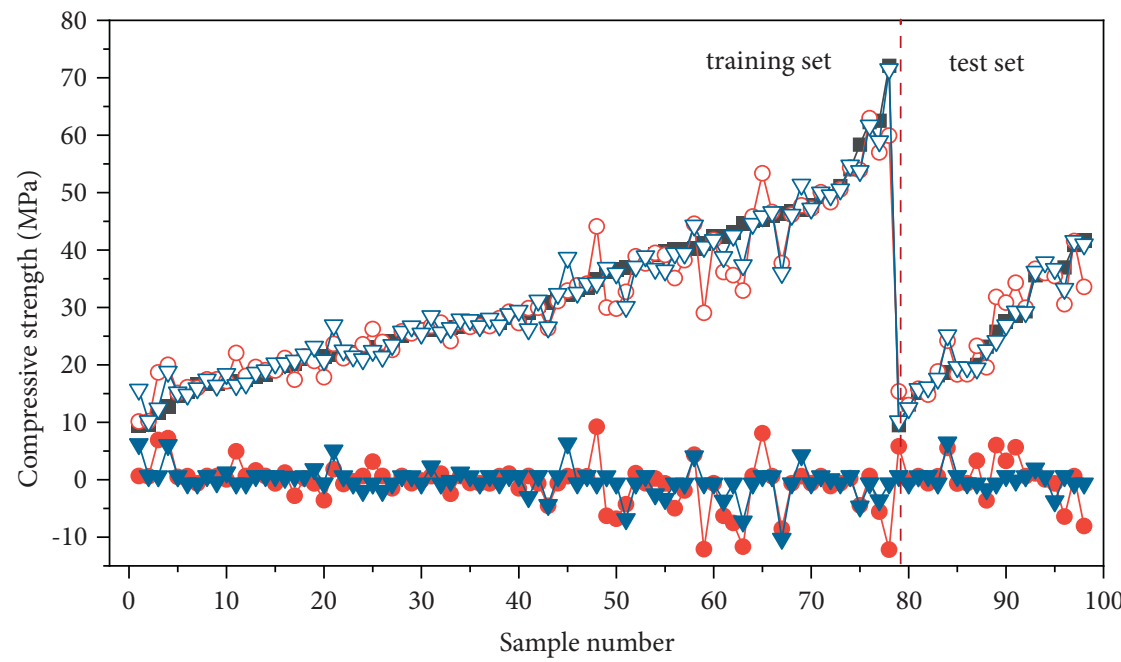

- Experimental value $\quad-\nabla$ - Predicted value of GS-SVR model

$-\mathrm{O}$ Predicted value of SVR model $\quad \longrightarrow$ Residual of GS-SVR model

$\rightarrow-$ Residual of SVR model

Figure 7: Comparison of predicted and actual values for two models.

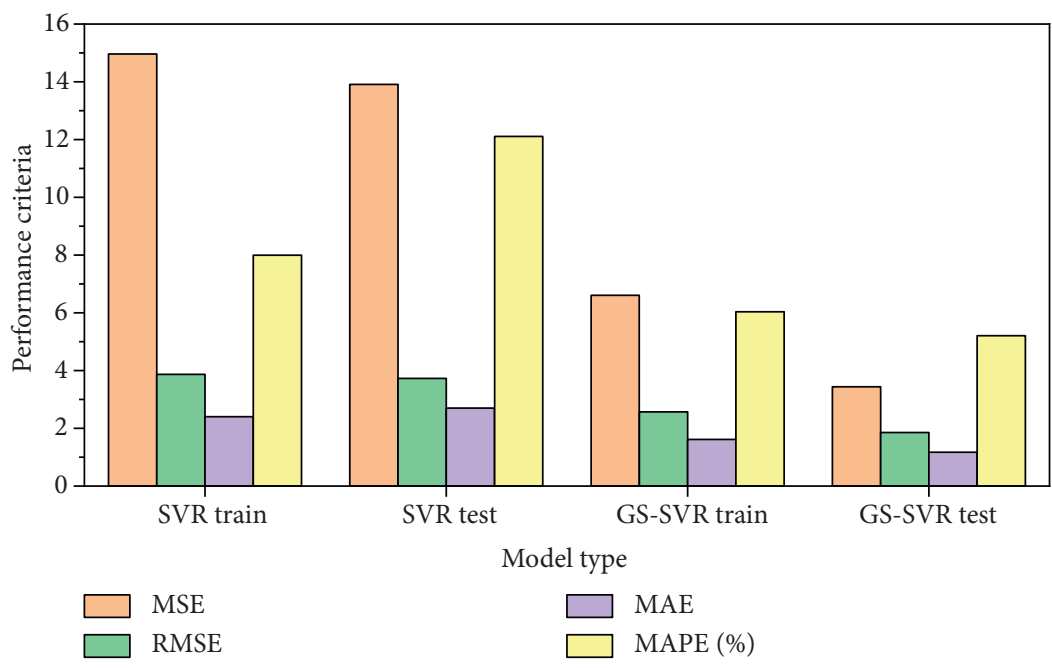

Figure 8: Performance criteria of the models.

TABLE 2: Deterministic values of configuration parameters.

\begin{tabular}{lcc}
\hline Variable & Unit & Value \\
\hline Cement & $\mathrm{kg} / \mathrm{m}^{3}$ & 230 \\
Fly ash & $\mathrm{kg} / \mathrm{m}^{3}$ & 125 \\
Water & $\mathrm{kg} / \mathrm{m}^{3}$ & 180 \\
Superplasticizer & $\mathrm{kg} / \mathrm{m}^{3}$ & 6 \\
Coarse aggregate & $\mathrm{kg} / \mathrm{m}^{3}$ & 1000 \\
Fine aggregate & $\mathrm{kg} / \mathrm{m}^{3}$ & 800 \\
Age & $\mathrm{d}$ & 28 \\
\hline
\end{tabular}

$$
C V=\frac{\sigma}{\mu},
$$

where $\sigma$ and $\mu$ are the standard deviation and mean values of the compressive strength, respectively. The distribution of compressive strength for the three randomness settings is shown in Figure 9.

As the randomness $S_{0}$ increases, the sensitivity of compressive strength for the three cases is higher. Additionally, among the seven variables, fly ash and coarse 


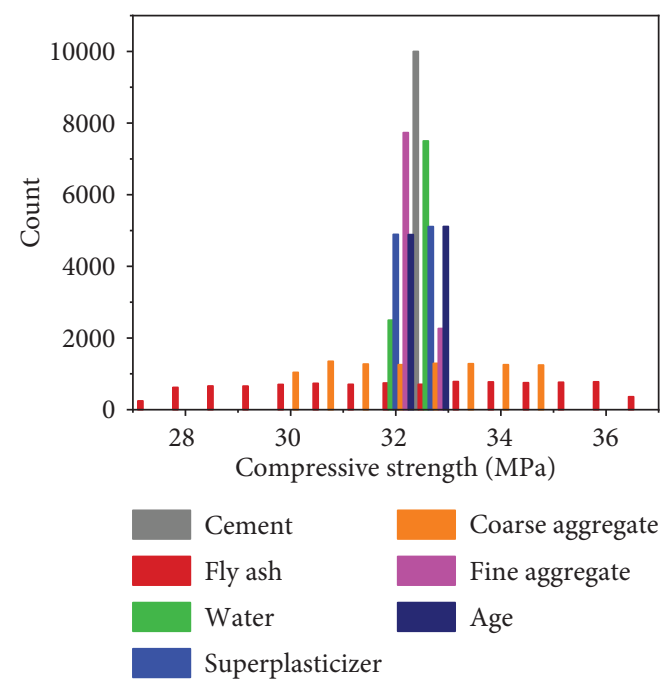

(a)

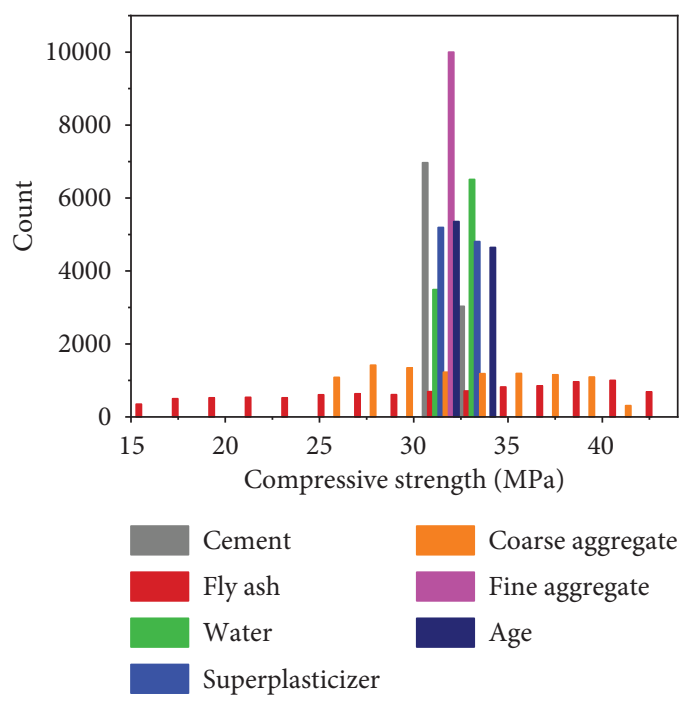

(c)

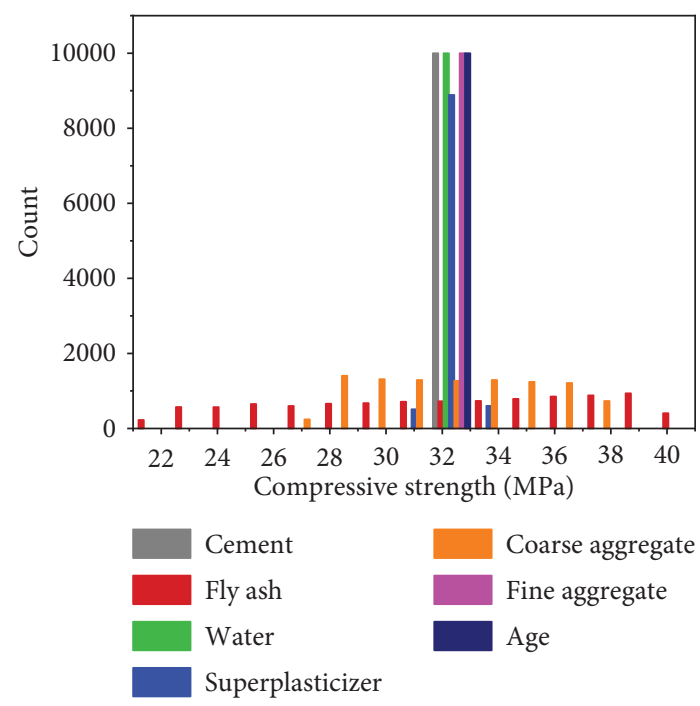

(b)

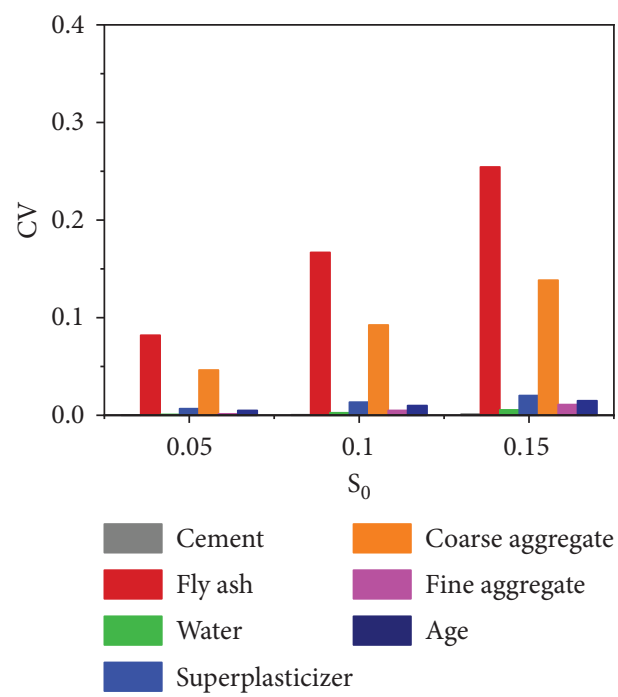

(d)

Figure 9: Distribution of the predicted values of compressive strength at different random degrees. (a) $S_{0}=0.05$. (b) $S_{0}=0.1$. (c) $S_{0}=0.15$. (d) Sensitivity.

aggregate resulted in a more discrete distribution of predicted compressive strength values, where fly ash has the greatest influence on the predicted uncertainty of compressive strength values. This insight might also be observed in Figure 9(d). Designers should take more attention to the design of mix design and compressive strength prediction of concrete with fly ash admixture in stochastic environments.

\section{GS-SVR Model-Based Interactive Graphical User Interface}

Nowadays, structural designers and engineers prefer to develop more robust and user-friendly software to gain wider applicability. In fact, to ensure that the model developed in this study is useful and practical and for ease of use, a graphical user interface (GUI) was compiled using MATLAB as shown in Figure 10. The whole interface is divided into two main parts: the optimization of hyperparameters and the prediction of output results with known input parameters. The operation of the GUI can be obtained by clicking on the Help menu, and the whole process consists of four main steps.

Step 1. Click the Initialize setting button to get the default values of the parameters; these values can also be modified manually.

Step 2. Click the Optimization button to obtain the optimal values of parameters $C$ and $g$

Step 3. Manually enter each input parameter

Step 4. Click the Predict button to get the final output value 


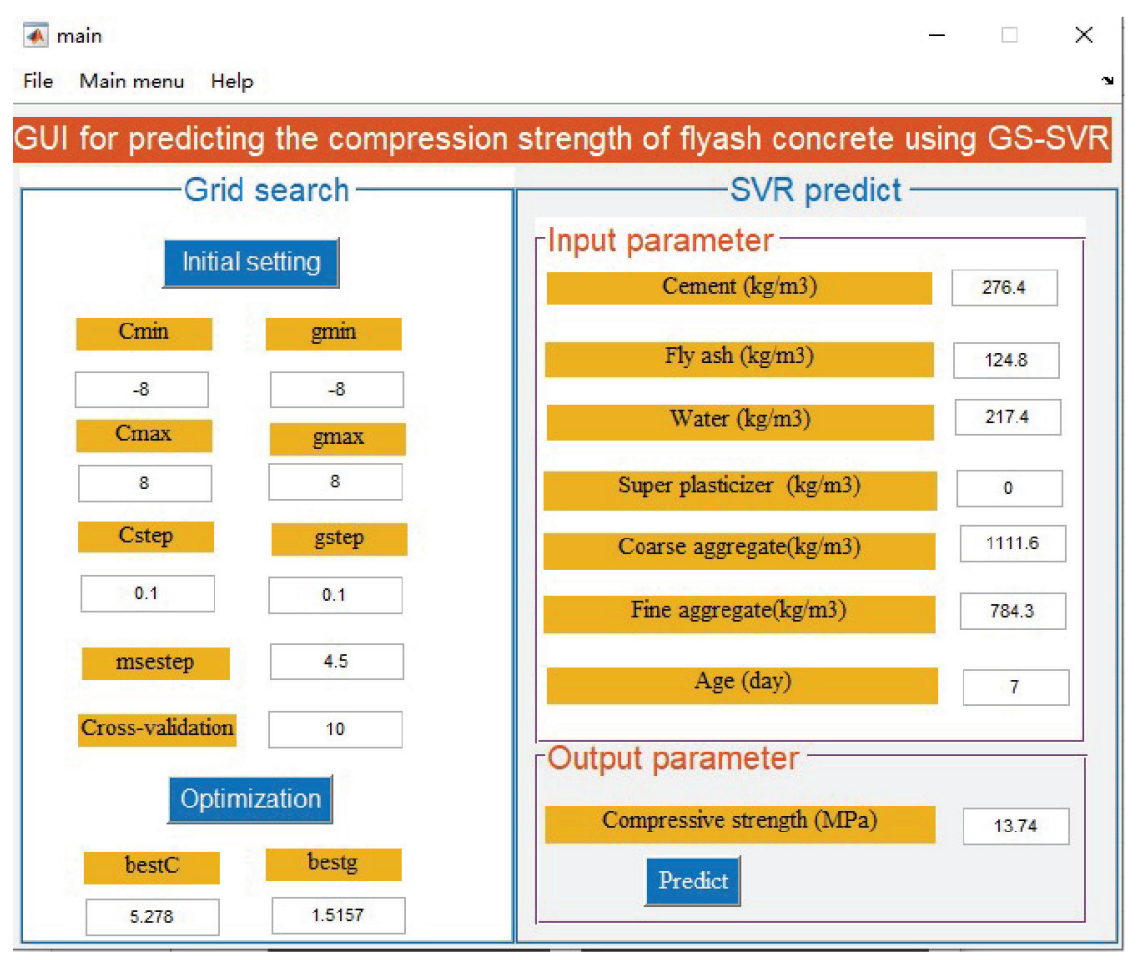

FIgURE 10: GS-SVR model-based interactive graphical user interface.

Finally, the compressive strength of fly ash concrete is displayed directly by clicking the Predict button. This GUI was developed mainly for the dataset used in this study, and future optimization of the user interface such as adding new datasets and other influencing parameters can be considered to make the model predictions more accurate.

\section{Conclusions}

In this work, a hybrid machine learning model GS-SVR was employed to predict the compressive strength of concrete with fly ash admixture and quantity the sensitivity of the compressive strength in the stochastic environment. The main findings are as follows.

(1) The proposed GS-SVR model can accurately capture the complex nonlinear relationship between the seven input parameters and the compressive strength of concrete with an accuracy $R$ of over $98 \%$ in both the training and test phases

(2) From the performance criteria, the prediction performance of the proposed model is better than that of the original SVR model, which is a promising candidate for evaluating the compressive strength of fly ash concrete

(3) In the stochastic environment, for the dataset used in this study, the compressive strength of concrete with fly ash admixture is most sensitive to fly ash, followed by the coarse aggregate, and the sensitivity to the other five input variables is weak.

(4) As the randomness of variables increases, the distribution range of compressive strength becomes wider and the dispersion becomes larger, and designers and engineers should pay more attention to the effect of random variation of fly ash and coarse aggregate on strength uncertainty.

(5) This study provides a new GUI that can be easily used to predict the compressive strength of fly ash concrete. This tool has been proven to be very successful, exhibiting very reliable predictions. Otherwise, it is idealistic to have some initial estimates of the outcomes before performing any extensive laboratory work or fieldwork.

This work also exhibits several limitations that need to be investigated in the future. First, the dataset used in this study is not large enough, and the effects of aggregate size and water reducing agent type on model prediction accuracy are not studied due to the lack of the dataset. Second, other machine learning algorithms or hybrid models can also be developed appropriately if higher prediction accuracy can be obtained. Finally, the current GUI is relatively simple and rough. As new datasets are added, the model needs to be retrained, and the GUI needs to be further updated and improved.

\section{Data Availability}

The dataset used to support the findings of this study and the GUI are available from the corresponding author upon request.

\section{Conflicts of Interest}

The authors declare that they have no conflicts of interest. 


\section{Acknowledgments}

The authors are grateful for the financial support from the Key Scientific and Technological Research Projects of Henan Province (222102210306).

\section{References}

[1] S. Barbhuiya, "Effects of fly ash and dolomite powder on the properties of self-compacting concrete," Construction and Building Materials, vol. 25, no. 8, pp. 3301-3305, 2011.

[2] A. Fuzail Hashmi, M. Shariq, and A. Baqi, "Flexural performance of high volume fly ash reinforced concrete beams and slabs," Structures, vol. 25, pp. 868-880, 2020.

[3] W. Ahmad, A. Ahmad, K. A. Ostrowski, F. Aslam, and P. Joyklad, "A scientometric review of waste material utilization in concrete for sustainable construction," Case Studies in Construction Materials, vol. 15, 2021.

[4] W. Ahmad, A. Ahmad, K. A. Ostrowski, F. Aslam, P. Joyklad, and P. Zajdel, "Sustainable approach of using sugarcane bagasse ash in cement-based composites: a systematic review," Case Studies in Construction Materials, vol. 15, 2021.

[5] Y. Hefni, Y. A. E. Zaher, and M. A. Wahab, "Influence of activation of fly ash on the mechanical properties of concrete," Construction and Building Materials, vol. 172, pp. 728-734, 2018.

[6] R. Hay and C. P. Ostertag, "New insights into the role of fly ash in mitigating alkali-silica reaction (ASR) in concrete," Cement and Concrete Research, vol. 144, 2021.

[7] S. Kang, Z. Lloyd, T. Kim, and M. T. Ley, "Predicting the compressive strength of fly ash concrete with the Particle model," Cement and Concrete Research, vol. 137, 2020.

[8] M. Sahmaran and V. C. Li, "Durability properties of microcracked ECC containing high volumes fly ash," Cement and Concrete Research, vol. 39, no. 11, pp. 1033-1043, 2009.

[9] P. Chopra, R. K. Sharma, M. Kumar, and T. Chopra, "Comparison of machine learning techniques for the prediction of compressive strength of concrete," Advances in Civil Engineering, vol. 2018, Article ID 5481705, 9 pages, 2018.

[10] J.-S. Chou and A.-D. Pham, "Enhanced artificial intelligence for ensemble approach to predicting high performance concrete compressive strength," Construction and Building Materials, vol. 49, pp. 554-563, 2013.

[11] F. Farooq, A. Akbar, R. A. Khushnood, W. L. B. Muhammad, S. K. U. Rehman, and M. F. Javed, "Experimental investigation of hybrid carbon nanotubes and graphite nanoplatelets on rheology, shrinkage, mechanical, and microstructure of SCCM," Materials, vol. 13, no. 1, 2020.

[12] A. F. Deifalla, A. G. Zapris, and C. E. Chalioris, "Multivariable regression strength model for steel fiber-reinforced concrete beams under torsion," Materials, vol. 14, no. 14, 2021.

[13] O. Sucharda, M. Pajak, T. Ponikiewski, and P. Konecny, "Identification of mechanical and fracture properties of selfcompacting concrete beams with different types of steel fibres using inverse analysis," Construction and Building Materials, vol. 138, pp. 263-275, 2017.

[14] M. Azimi-Pour, H. Eskandari-Naddaf, and A. Pakzad, "Linear and non-linear SVM prediction for fresh properties and compressive strength of high volume fly ash self-compacting concrete," Construction and Building Materials, vol. 230, 2020.

[15] J. Zhang, G. Ma, Y. Huang, J. Sun, F. Aslani, and B. Nener, "Modelling uniaxial compressive strength of lightweight selfcompacting concrete using random forest regression," Construction and Building Materials, vol. 210, pp. 713-719, 2019.
[16] E. M. Golafshani and A. Ashour, "Prediction of self-compacting concrete elastic modulus using two symbolic regression techniques," Automation in Construction, vol. 64, pp. 7-19, 2016.

[17] P. Saha, P. Debnath, and P. Thomas, "Prediction of fresh and hardened properties of self-compacting concrete using support vector regression approach," Neural Computing \& Applications, vol. 32, no. 12, pp. 7995-8010, 2020.

[18] P. Neira, L. Bennun, M. Pradena, and J. Gomez, "Prediction of concrete compressive strength through artificial neural networks," Građevinar, vol. 72, no. 7, pp. 585-592, 2020.

[19] S. Kostic and D. Vasovic, "Prediction model for compressive strength of basic concrete mixture using artificial neural networks," Neural Computing \& Applications, vol. 26, no. 5, pp. 1005-1024, 2015.

[20] Z. H. Duan, S. C. Kou, and C. S. Poon, "Prediction of compressive strength of recycled aggregate concrete using artificial neural networks," Construction and Building Materials, vol. 40, pp. 1200-1206, 2013.

[21] P. Parthiban and J. Karthikeyan, "Artificial neural network to predict the compressive strength of semilightweight concrete containing ultrafine GGBS," Journal of Testing and Evaluation, vol. 48, no. 2, pp. 795-810, 2019.

[22] J. Zhang, J. Xu, C. Liu, and J. Zheng, "Prediction of rubber fiber concrete strength using extreme learning machine," Frontiers in Materials, vol. 7, no. 465, 2021.

[23] Y. R. Wang, Y. L. Lu, and D. L. Chiang, "Adapting artificial intelligence to improve in situ concrete compressive strength estimations in rebound hammer tests," Frontiers in Materials, vol. 7 , no. $365,2020$.

[24] H. B. Ly, T. A. Nguyen, H. V. T. Mai, and V. Q. Tran, "Development of deep neural network model to predict the compressive strength of rubber concrete," Construction and Building Materials, vol. 301, 2021.

[25] Q. Han, C. Gui, J. Xu, and G. Lacidogna, “A generalized method to predict the compressive strength of high-performance concrete by improved random forest algorithm," Construction and Building Materials, vol. 226, pp. 734-742, 2019.

[26] F. Farooq, S. Czarnecki, P. Niewiadomski et al., "A comparative study for the prediction of the compressive strength of self-compacting concrete modified with fly ash," Materials, vol. 14 , no. $17,2021$.

[27] X. Zhang, M. Z. Akber, and W. Zheng, "Prediction of sevenday compressive strength of field concrete," Construction and Building Materials, vol. 305, Article ID 124604, 2021.

[28] K. T. Nguyen, Q. D. Nguyen, T. A. Le, J. Shin, and K. Lee, "Analyzing the compressive strength of green fly ash based geopolymer concrete using experiment and machine learning approaches," Construction and Building Materials, vol. 247, Article ID 118581, 2020.

[29] A. K. Al-Shamiri, J. H. Kim, T.-F. Yuan, and Y. S. Yoon, "Modeling the compressive strength of high-strength concrete: an extreme learning approach," Construction and Building Materials, vol. 208, pp. 204-219, 2019.

[30] W. Jiang, Y. J. Xie, W. X. Li, J. X. Wu, and G. C. Long, "Prediction of the splitting tensile strength of the bonding interface by combining the support vector machine with the particle swarm optimization algorithm," Engineering Structures, vol. 230, 2021.

[31] C.-C. Chang and C.-J. Lin, "Training v-support vector regression: theory and algorithms," Neural Computation, vol. 14, no. 8, pp. 1959-1977, 2002. 
[32] C.-C. Chang and C.-J. Lin, "LIBSVM: a library for support vector machines," ACM Transactions on Intelligent Systems and Technology, vol. 2, no. 3, Article ID 27, 2011.

[33] Y. R. Liu, L. Wang, and K. X. Gu, “A support vector regression (SVR)-based method for dynamic load identification using heterogeneous responses under interval uncertainties," $A p$ plied Soft Computing, vol. 110, 2021.

[34] M. Hadzima-Nyarko, E. K. Nyarko, N. Ademovic, I. Milicevic, and T. K. Sipos, "Modelling the influence of waste rubber on compressive strength of concrete by artificial neural networks," Materials, vol. 12, no. 4, 2019.

[35] M. S. T. Nguyen, M. C. Trinh, and S. E. Kim, "Uncertainty quantification of ultimate compressive strength of CCFST columns using hybrid machine learning model," Engineering with Computers, 2021.

[36] M. S. T. Nguyen and S. E. Kim, "A hybrid machine learning approach in prediction and uncertainty quantification of ultimate compressive strength of RCFST columns," Construction and Building Materials, vol. 302, 2021. 